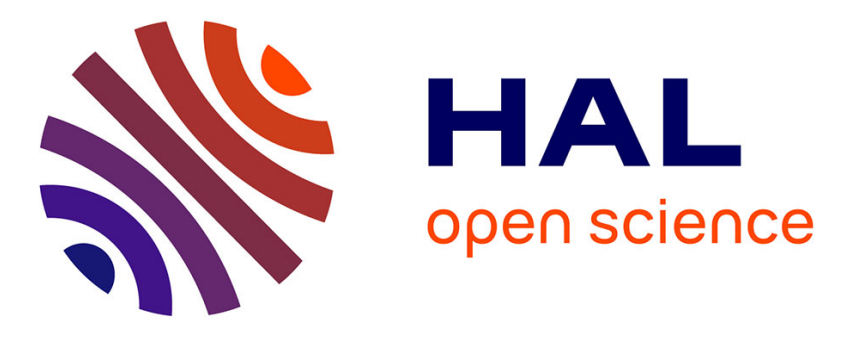

\title{
Near-field Optical Images of Subwavelength Annular Aperture Arrays Exhibiting an Extraordinary Transmission
}

Y. Poujet, J. Salvi, F.I Baida, D. van Labeke, A. Perentes, C. Santschi, P. Hoffmann

\section{To cite this version:}

Y. Poujet, J. Salvi, F.I Baida, D. van Labeke, A. Perentes, et al.. Near-field Optical Images of Subwavelength Annular Aperture Arrays Exhibiting an Extraordinary Transmission. Journal of Microscopy, 2008, 229 (2), pp.203-209. 10.1111/j.1365-2818.2008.01887.x . hal-00194818

\section{HAL Id: hal-00194818 https://hal.science/hal-00194818}

Submitted on 16 Apr 2021

HAL is a multi-disciplinary open access archive for the deposit and dissemination of scientific research documents, whether they are published or not. The documents may come from teaching and research institutions in France or abroad, or from public or private research centers.
L'archive ouverte pluridisciplinaire HAL, est destinée au dépôt et à la diffusion de documents scientifiques de niveau recherche, publiés ou non, émanant des établissements d'enseignement et de recherche français ou étrangers, des laboratoires publics ou privés.

\section{(c)(1)}

Distributed under a Creative Commons Attribution| 4.0 International License 


\title{
Near-field optical images of subwavelength annular aperture arrays exhibiting an extraordinary transmission
}

\author{
Y. POUJET ${ }^{*}$, J.SALVI ${ }^{*}$, F.I.BAIDA ${ }^{*}$, D. VAN LABEKE ${ }^{*}$, A. PERENTES ${ }^{\dagger}$, C. SANTSCHI $^{\dagger} \&$ P. HOFFMANN $^{\dagger}$
}

*Institut FEMTO-ST UMR 6174, Département d'Optique P.M. Duffieux, Université de

Franche-Comté, 16 route de Gray, 25030 Besançon Cedex, France

$\dagger$ IOA, École Polytechnique Fédérale de Lausanne, CH-1015 Lausanne, Switzerland

Key words. annular aperture arrays, extraordinary transmission, guided mode, RSTOM, SNOM.

\begin{abstract}
Summary
In this paper, we present near-field optical images of nanostructures exhibiting an extraordinary transmission. These structures consist of annular aperture arrays engraved in a metallic film: they are quite promising structures for nanophotonics because of their high transmission directly linked to a guided mode mediated by each annular aperture. We first briefly explain our fabrication process (focused ion beam milling), then we expose the experimental setup of the near-field optical microscope working both in reflection and transmission modes. For the reflection mode, the 'coffee-bean' structure of the electromagnetic field predicted by the theory has been quite well reproduced. For the transmission mode, we present preliminary experimental results concerning the influence of the wavelength and the polarization of the incident beam on the obtained near-field images.
\end{abstract}

\section{Introduction}

It is well known now that light can substantially pass through subwavelength nano-apertures in a thick metallic film (Ebbesen et al., 1998; Martin-Moreno et al., 2001), unlike the hasty predictions that can be deduced from the 'classical' theory (Bethe, 1944; Bouwkamp, 1954). A transmission peak in the visible range can be obtained and it reaches 10\% (Ebbesen et al., 1998): this transmission is due to a surface plasmon resonance mediated by the nanostructures (Popov et al., 2000). Many applications made the most of this phenomenon in different domains: fluorescence (Liu \& Blair, 2003), nanophotonics (Shinada et al., 2003) or chemical sensors (Brolo et al., 2004). In order to increase

Correspondence to: Y. Poujet. Tel: +33 3816664 08; fax: +33 3816664 23; e-mail: yannick.poujet@univ-fcomte.fr the light transmission in the visible range, Baida and Van Labeke proposed in 2002 a new geometry that consists of annular apertures arranged in a bi-periodic array (Baida \& Van Labeke, 2002). This structure was named AAA for annular aperture array. A similar idea has been already developed by Roberts and Mac Phedran in the case of perfect conductors (Roberts \& McPhedran, 1988). For specific dimensions, the AAA can theoretically lead to a transmission peak of around $90 \%$ in the visible range of the spectrum (Baida \& Van Labeke, 2003). This high value of the transmission is directly connected to the existence of a guided mode inside the coaxial apertures (Baida et al., 2004; Baida et al., 2006). The aim of this paper is to study these kind of nanostructures in the near-field domain. After having explained our fabrication process and our calculation method, we present experimental results obtained with a reflection scanning tunneling optical microscope (RSTOM). As already mentioned in a previous work (Poujet et al., 2006), the same 'coffee-bean' light distribution is observed with other geometries of the AAA. Finally, original experimental near-field images obtained with a scanning nearfield optical microscope (SNOM) working in transmission mode are presented for different polarizations and wavelengths.

\section{Fabrication process}

The fabrication process is the same as described elsewhere (Elsner \& Meyer, 2001). The substrate consists of a $120-\mu \mathrm{m}$ thick fused silica cover slide, on which a $5-\mathrm{nm}$ adhesion layer (titanium or chromium) and a metallic film (silver or gold) are evaporated. A low evaporation rate prevents from grain formation onto the metallic layer. The surface roughness, measured by atomic force microscopy, is then about $2 \mathrm{~nm}$. A NOVA 600 dual-beam system from FEI Company with installed focused ion and electron beam was used for the milling experiments. Compared to focused electron 


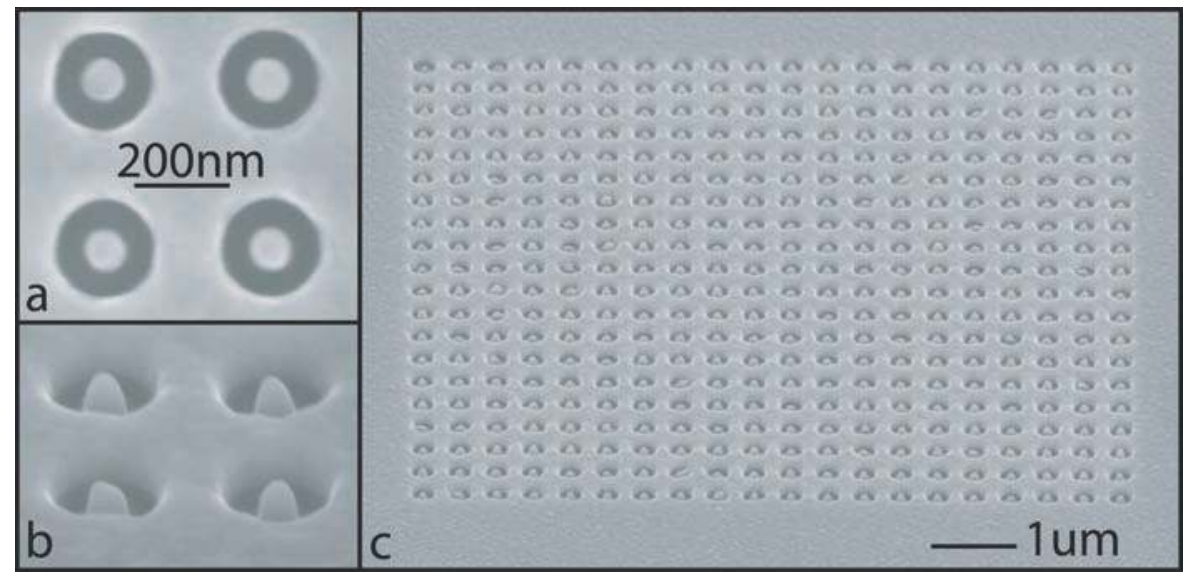

Fig. 1. Example of an AAA (diameters: $200 / 100 \mathrm{~nm}$ and period: $350 \mathrm{~nm}$ ) made by FIB milling: (a) top view of two periods, (b) view with a $52^{\circ}$ tilt and (c) view of the whole matrix.

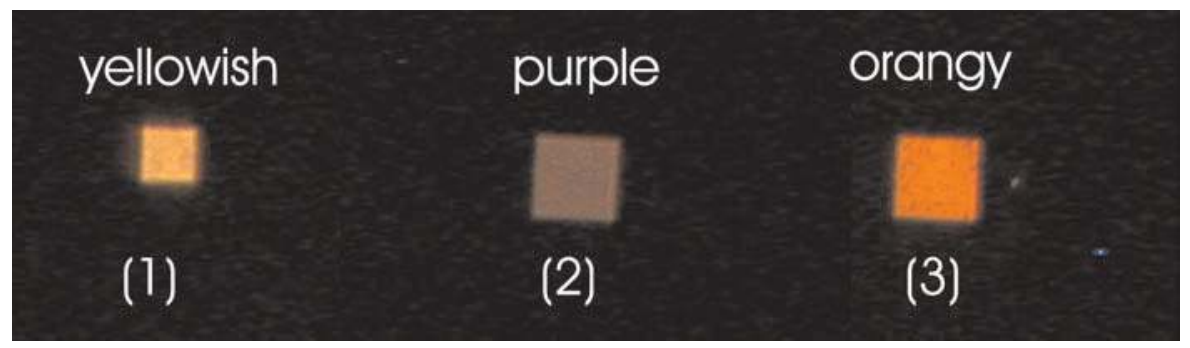

Fig. 2. Far field image of three different AAAs in real colours obtained by conventional microscope working in transmission mode. The AAAs are engraved on a 100-nm thick silver layer. The inner diameter $\left(d_{i}\right)$, the outer diameter $\left(d_{o}\right)$ and the period $p$ are $d_{i}=100 \mathrm{~nm}, d_{o}=200 \mathrm{~nm}, p=350 \mathrm{~nm}$ for matrix (1) $\left(7 \times 7 \mu \mathrm{m}^{2}\right) ; d_{i}=230 \mathrm{~nm}, d_{o}=330 \mathrm{~nm}, p=500 \mathrm{~nm}$ for matrix $(2)\left(10 \times 10 \mu \mathrm{m}^{2}\right)$ and $d_{i}=100 \mathrm{~nm}, d_{o}=200 \mathrm{~nm}, p=500 \mathrm{~nm}$ for matrix $(3)$ $\left(10 \times 10 \mu \mathrm{m}^{2}\right)$.

beam (FEB) lithography, focused ion beam (FIB) milling is an adequate technique for rapid fabrication of prototype optical nanostructures (Perentes et al., 2005). Nanoscale structures are obtainable with perfectly tuned FIBs, and parameters such as dwell time, overlap, astigmatism and focus are determining for the final structure quality. The fine tuning of the beam focus and the stigmator was done by milling test structures and painstaking readjustments have been carried out prior to the realization of the arrays. The FIB operated at $30 \mathrm{keV}$ and $10 \mathrm{pA}$ (beam diameter around $12 \mathrm{~nm}$ ) for all structures. An overlap of $50 \%$ in the $\mathrm{x}$ - and $\mathrm{y}$-directions has been introduced to ensure a homogeneous milling. A short dwell time (200 ns) and a large number of passes have been chosen to minimize the redeposition effects. FIB drift due to charging on the underlaying silica insulating substrate was minimized by irradiating the surface using the electron beam. The adequate ion dose to reach the designated depth and to investigate the size and the shape was determined by cross-cutting of the test structures after covering them with a platinum-containing material using FEB-induced deposition. This layer protects the structure of interest during FIB milling and offers a better contrast for the subsequent scanning electronic microscope imaging.
The quality of the final result is checked by imaging the structure thanks to a scanning electron microscope (see Fig. 1). A conventional optical microscope (REICHERT-JUNG) has been used to observe three different AAAs made in the same silver layer. Figure 2 shows the three far field images obtained in transmission mode (magnifying $=\times 50$ ). The whole sample was illuminated by a thermal white light source. As already shown elsewhere (Barnes et al., 2003; Kanamori et al., 2006), one can notice the spectral filtering possibilities of our structure.

The far field response of such a structure depends both on the geometrical parameters of one aperture and on the period of the whole array. More quantitative experiments are in progress with these samples made in silver but we already showed and compared theoretical and experimental transmissions in the case of AAAs in gold (Salvi et al., 2005).

Let us note here that, in the following, the presented experimental data are often compared with theoretical ones. These latest data are found through a finite difference time domain (FDTD) method that directly solves Maxwell's equations. It is a 3D home-made code where space and time are both discretized. In order to describe the fine features of the structure, a non-uniform meshing is implemented. This 


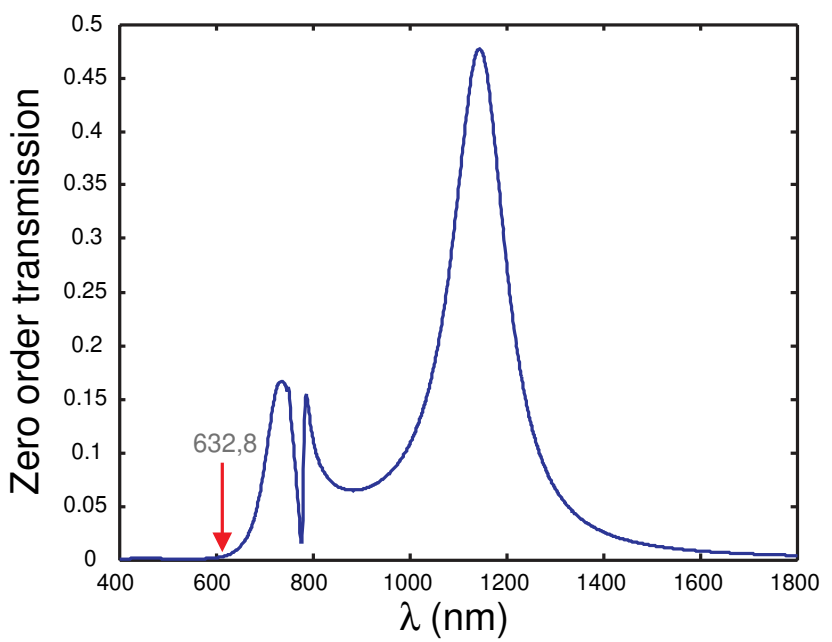

Fig. 3. Theoretical spectral response of the AAA studied in near-field optics (reflection mode): $d_{i}=200 \mathrm{~nm}, d_{o}=280 \mathrm{~nm}, p=500 \mathrm{~nm}$.

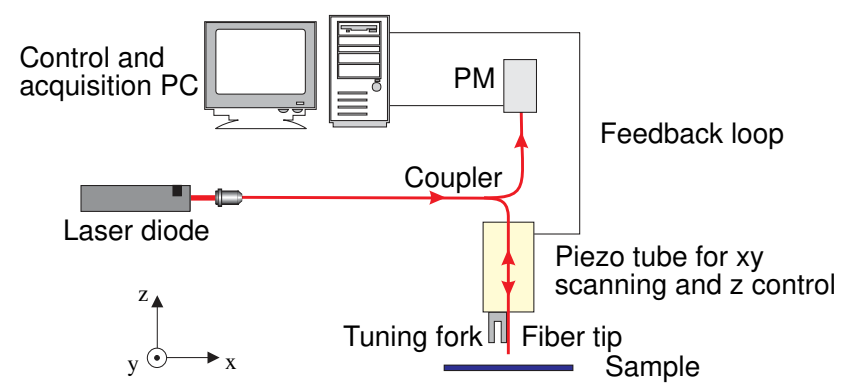

Fig. 4. Scheme of the experimental RSTOM setup.

leads to a decrease in the total number of nodes (spatial steps: $\Delta=5 \mathrm{~nm}$ for the metallic film and $\Delta=25 \mathrm{~nm}$ for the rest of the calculation window). This mesh variation is carried out gradually to avoid parasitical reflections. In addition, the Berenger's perfectly matched layers are incorporated into this code to cancel the reflection on the external edges of the computational window. More details on the numerical simulations can be found in references (Baida \& Van Labeke, 2002; Poujet et al., 2006). All the theoretical results presented in this paper are made with this code.

\section{Near-field optics: reflection mode}

The AAAs under study in this section have been made by FIB milling in a 100-nm thick gold layer deposited on a glass substrate with a thin chromium adhesion layer $(5 \mathrm{~nm})$. The period of the structure is $500 \mathrm{~nm}$, the inner diameter of the coaxes is $200 \mathrm{~nm}$ and the outer diameter is $280 \mathrm{~nm}$. It's theoretical spectral response is plotted in Fig. 3.

The near-field experiments are achieved with a commercial SNOM (SMENA model). The experimental setup is described in Fig. 4 . The sample is illuminated at $\lambda=632.8 \mathrm{~nm}$ thanks to a dielectric tip. The light diffracted by the sample is detected by the same tip and a coupler is used to separate the incident and the backward signals. The near-field signal is then recorded by a photomultiplier (PM) and analyzed by an appropriate software.

The shear-force and the near-field optical images are shown in Fig. 5: the optical image is blurred by fringes. The origin of these fringes is not completely understood but they are very common in this kind of experiments. The interferences may be due to the interaction between the incident and the reflected light, the coupling between the tip and the sample and/or the tip shape or size. This image has been filtered in order to eliminate the fringes. This filtering simply consists in a 2D fast fourier transform (FFT), which allows us to cancel the spatial frequency corresponding to the fringes by means of a binary filtering function. A 'coffee-bean'-like distribution of the electric field then becomes apparent, as shown in Fig. 6(a). The zone marked with a white square is magnified and displayed in Fig. 6(b). We also marked with white circles the position of some annular cavities deduced from the simultaneously recorded shear-force image. This result demonstrates, if necessary, the connection between the annular cavity geometry and the two-lobe light distribution. A similar result has been already obtained with another AAA having different dimensions (Poujet et al., 2006).

This two-lobe structure is typical of the $\mathrm{TE}_{11}$-like mode (Baida et al., 2004, 2006). The theoretical calculation (3DFDTD) presents the same distribution of the electromagnetic field (see Fig. 6c). This image corresponds to the square modulus of the transversal components of the electric field recorded $30 \mathrm{~nm}$ above the structure. The polarization of the incident field is along the two maxima of light. There is a good agreement between the experimental and the theoretical images if we consider that the dielectric tip is thin enough to act as a dipole that only extracts the tangential components of the electric field even if the normal one is larger (Van Labeke \& Barchiesi, 1993; Grosjean \& Courjon, 2003). Note that the illuminating wavelength is out of the high transmission region of the spectrum as shown in Fig. 3 (red arrow). So, the cavity mode cannot propagate inside the annular aperture and it is completely reflected: it just settles at the entry of the cavity and its intensity exponentially decays when penetrating inside the aperture.

\section{Near-field optics: transmission mode}

The near-field optical images in transmission mode have been carried out with an AAA made in a 125 -nm thick silver layer. The period is $500 \mathrm{~nm}$, the inner ring and the outer ring diameters are $100, \mathrm{~nm}$ and $200 \mathrm{~nm}$, respectively. As previously described, the AAAs have been made by following the same FIB milling process. To our knowledge, this is the first study concerning the polarization effect and the wavelength influence for such a structure. As shown in Fig. 7, a powerful white light source generated by a pulsed laser at $\lambda=532 \mathrm{~nm}$ is focused into a dispersion shifted fiber (Mussot et al., 2003). A microscope objective collimates the emerging beam, which 


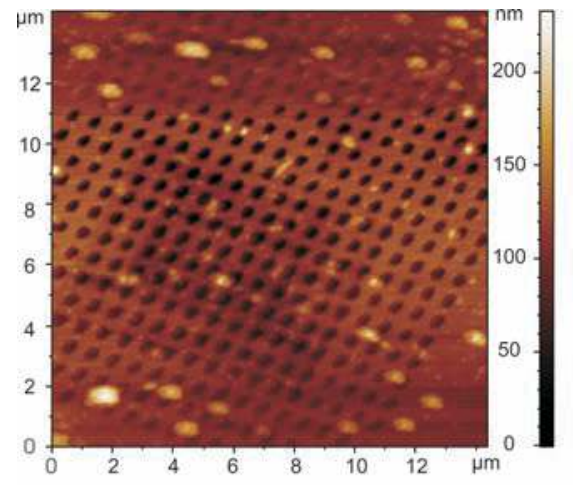

Shear-force image

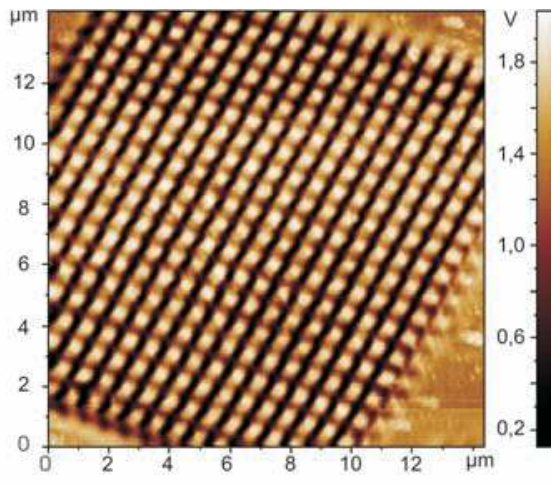

Optical image

Fig. 5. Shear-force and optical images $\left(14 \times 14 \mu \mathrm{m}^{2}\right)$ of an AAA in a 100-nm thick gold film. The inner diameter is $200 \mathrm{~nm}$, the outer diameter $280 \mathrm{~nm}$ and the period $500 \mathrm{~nm}$.

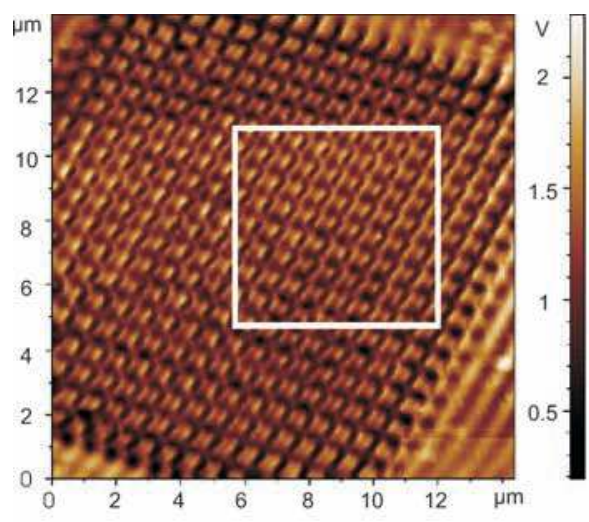

(a) Filtered optical image

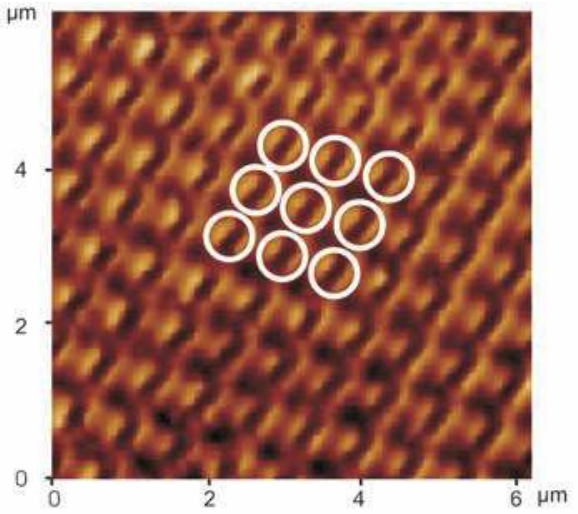

(b) Zoom in of image (a)

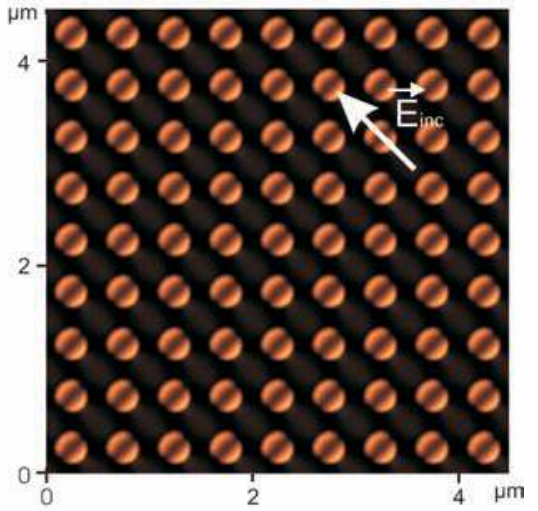

(c) Theoretical response

Fig. 6. (a) Filtered optical image of the near-field optical image in Fig. 5; (b) closeup of the white square in picture (a) $\left(6 \times 6 \mu \mathrm{m}^{2}\right)$ and (c) theoretical response obtained by FDTD calculation (polarization in the upper right corner).

illuminates the sample. The polarization direction is controlled with a Glan-Taylor polarizer and an interferential filter allows us to choose the incident wavelength. The images are recorded with the same SMENA commercial SNOM using a dielectric tip.

Shear-force and optical images of the same sample but for two different polarization directions are shown in Fig. 8. The theoretical spectral response of this sample is plotted in Fig. 9. The shear-force images that give the topography of the sample are identical: they correspond exactly to the same area of the sample. The optical images are recorded at $\lambda=622 \mathrm{~nm}$. The range of intensities is the same for both optical images so that we can quantitatively compare the obtained intensities. The two polarizations used in the experiments are first along the y-axis (top image in Fig. 8) and second at $45^{\circ}$ from the y-axis (bottom image in Fig. 8). The 'coffeebean' light distribution is not resolved in these images. Nevertheless the two optical images are very similar, which is in good agreement with theoretical findings of reference

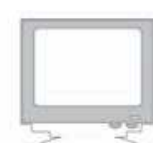

Control and acquisition PC
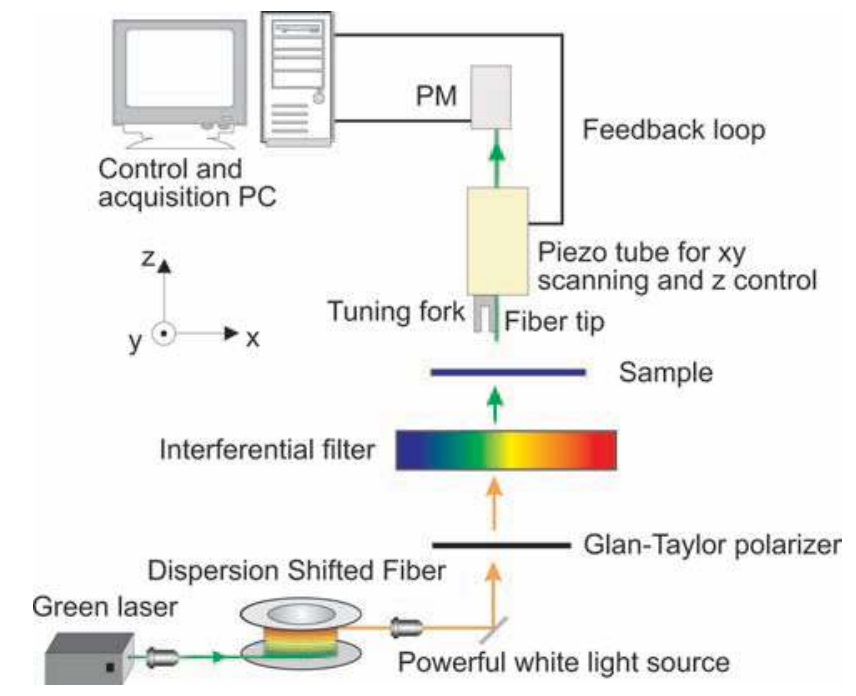

Feedback loop

Fig. 7. Scheme of the experimental SNOM setup. 

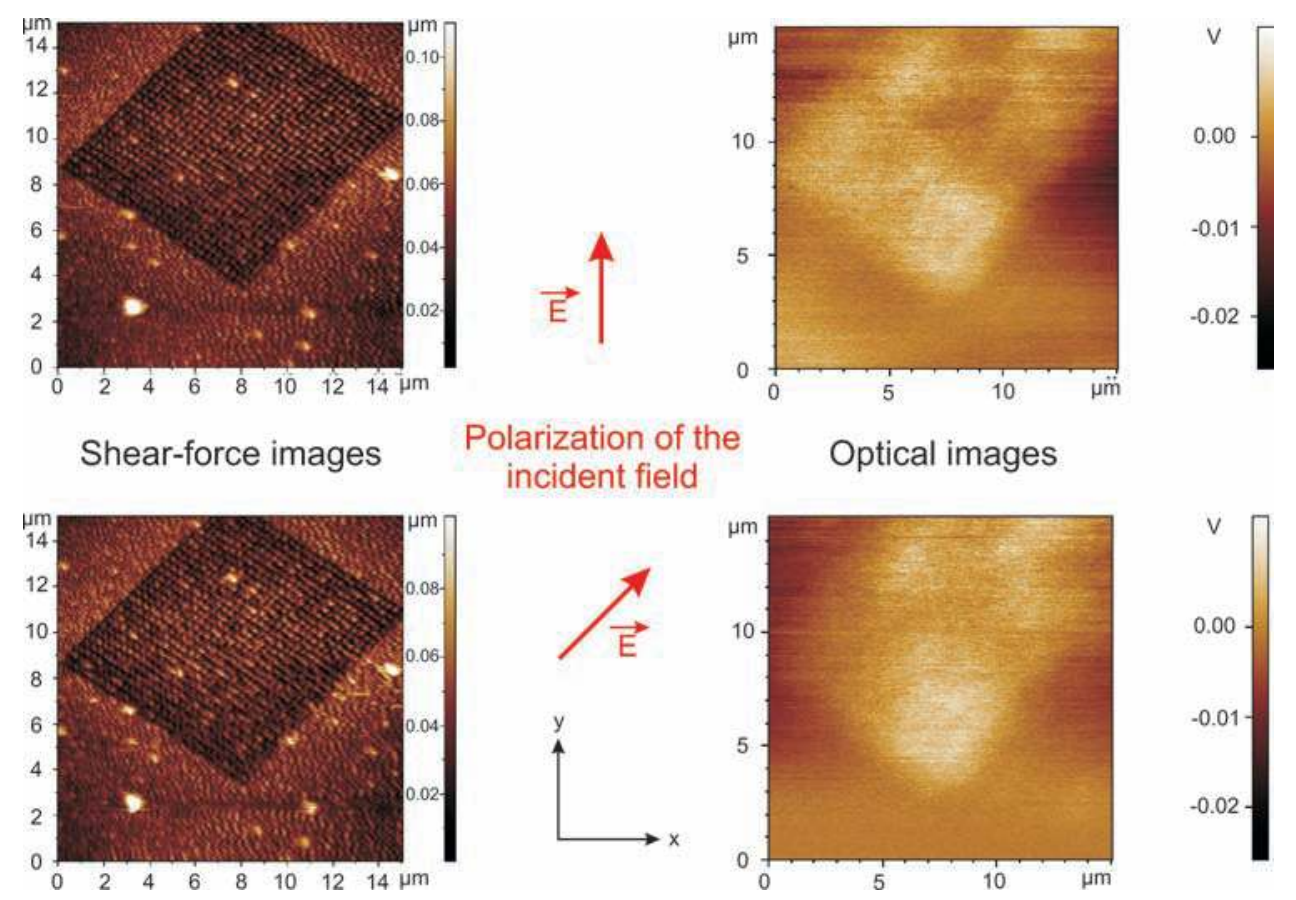

Fig. 8. Topographic and near-field optical images $\left(15 \times 15 \mu \mathrm{m}^{2}\right)$ with the same wavelength $\lambda=622 \mathrm{~nm}$ at different polarizations.

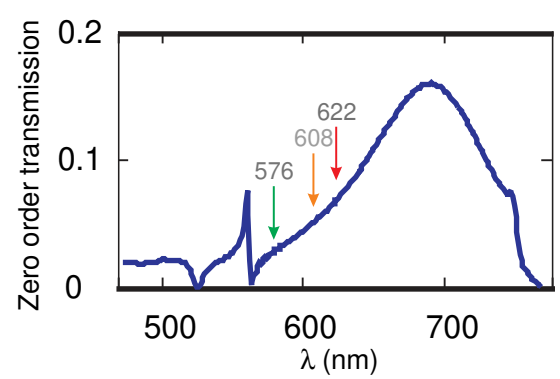

Fig. 9. Theoretical transmission of the studied AAA. The arrows mark the wavelengths used in the experiment.

(Baida \& Van Labeke, 2002). This is mainly due to the fact that the cylindrical symmetry of the annular apertures is insensitive to the polarization direction.

Near-field optical responses of one AAA matrix at different wavelengths $(576 \mathrm{~nm}, 608 \mathrm{~nm}$ and $622 \mathrm{~nm}$, see Fig. 9) have been also recorded in Fig. 10. The polarization is kept along the y-axis for all images. The intensity of each image is normalized to the source spectrum because the incident power can differ from one wavelength to another (Fig. 11). The grey level scale is the same for each optical image of Fig. 10. First, there is some light outside the AAA and the expected 'coffee-bean' distribution of the electric field is not resolved: as it is mentioned above, it may be due to the tip shape and/or to surface plasmons excited besides/on the matrix. Moreover, the transmitted zero order can also smear our SNOM images. Using a metallized tip could increase the resolution of our SNOM, preventing in this way the detection of the transmitted far field: the 'coffee-bean' structure of the near field could be then resolved. Second, we observe that the average value of the detected light intensity decreases when the wavelength increases. This behaviour is still unclear because recent theoretical calculations (not presented here) show a contradictory evolution for the intensity. More experimental and theoretical works are still in progress to completely understand these results.

\section{Conclusion}

In this paper, we qualitatively show the ability of AAAs to filter the light. More quantitative spectra are in progress to validate the high transmission in the visible range $(80 \%)$. However, the main part of this paper is dedicated, to our knowledge, to the first experimental near-field studies of such nanostructures. For the reflection mode, the experiment is in good accordance with the theory: we experimentally highlight the existence of the $\mathrm{TE}_{11}$-like mode by retrieving the typical two-lobe structure of light intensity above the coaxes. For the images obtained in transmission mode, we demonstrate that the polarization has no significant influence on the nearfield response. Nonetheless, the 'coffee-bean' structure of the electromagnetic field has not been resolved. In addition, the variation of the detected intensity according to the wavelength does not fulfil the theoretical predictions. These works are still in progress in order to clarify all the underlying physical phenomena. 

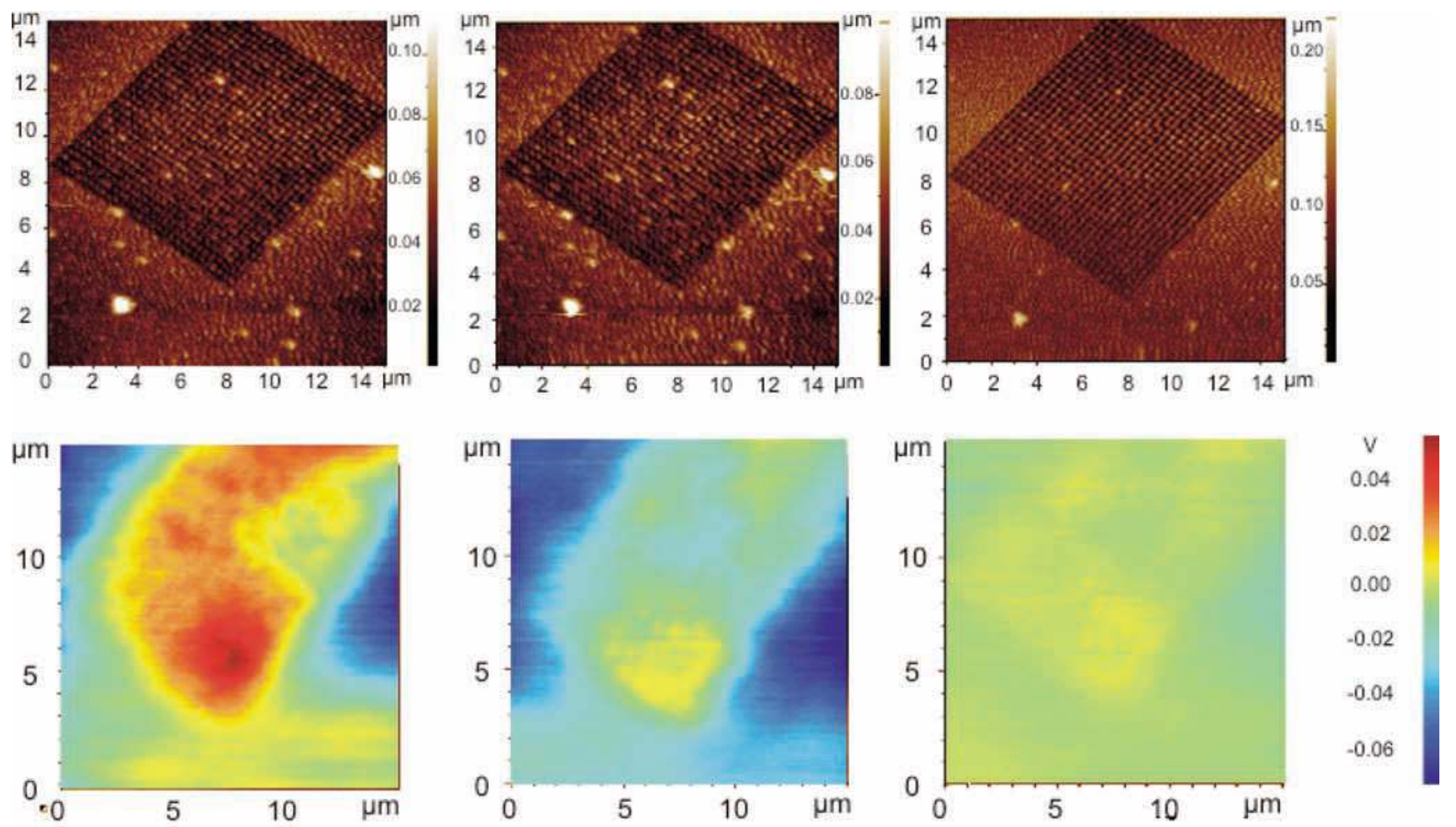

Fig. 10. Topographic and near-field optical images $\left(15 \times 15 \mu \mathrm{m}^{2}\right)$ with the same polarization at different incident wavelengths $\lambda$; from left to right $\lambda=$ $576 \mathrm{~nm}, 608 \mathrm{~nm}$ and $622 \mathrm{~nm}$.

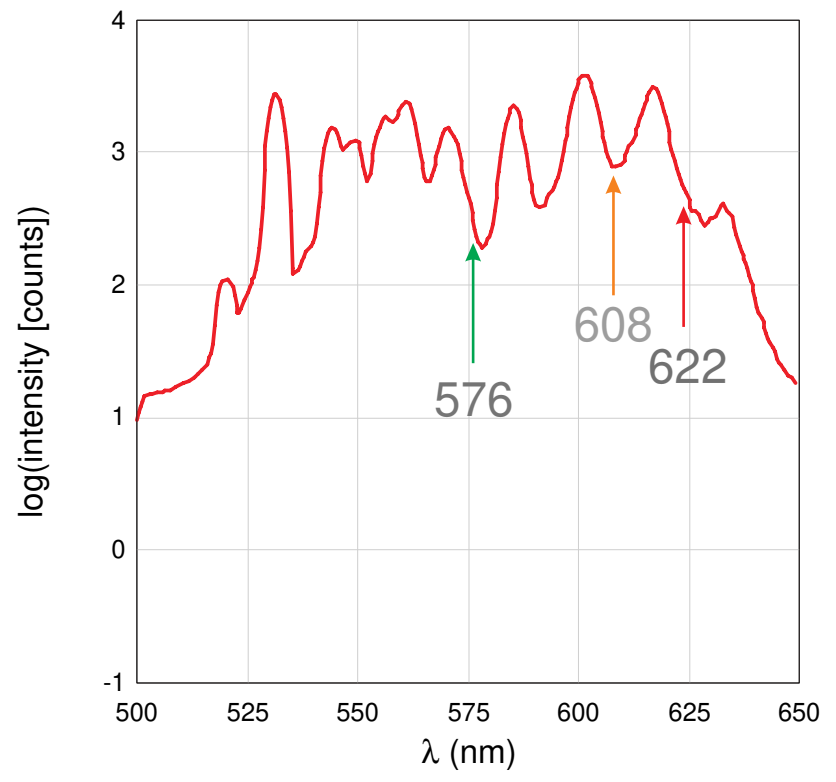

Fig. 11. Source spectrum. The arrows mark the wavelengths used in the experiment.

\section{Acknowledgments}

The authors thank the "Laboratoire Européen Associé FrancoSuisse" and the Network of Excellence in Micro-Optics for financial support.

\section{References}

Baida, F. \& Van Labeke, D. (2002) Light transmission by subwavelength annular aperture arrays in metallic films. Opt. Commun. 209, 17-22.

Baida, F. \& Van Labeke, D. (2003) Three-dimensional structures for enhanced transmission through a metallic film: annular aperture arrays. Phys. Rev. B 67, 1553141-7.

Baida, F., Van Labeke, D., Granet, G., Moreau, A. \& Belkhir, A. (2004) Origin of the super-enhanced light transmission through a 2-d metallic annular aperture array: a study of photonic bands. Appl. Phys. B 79, $1-8$.

Baida, F.I., Belkhir, A., Van Labeke, D. \& Lamrous, O. (2006) Subwavelength metallic coaxial waveguides in the optical range: role of the plasmonic modes. Phys. Rev. B 74, 205-419.

Barnes, W., Dereux, A. \& Ebbesen, T. (2003) Surface plasmon subwavelength optics. Nature 424, 824-830. Article sur les plasmons pour expliquer la transmission exalte.

Bethe, H. (1944) Theory of diffraction by small holes. Phys. Rev. 66, 163182.

Bouwkamp, C.J. (1954) Diffraction theory. Rep. Prog. Phys. 27, 35-100. Brolo, A., Gordon, R., Leathem, B. \& Kavanagh, K. (2004) Surface plasmon sensor based on the enhanced light transmission through arrays of nanoholes in gold films. Langmuir. 20, 4813-4815.

Ebbesen, T.W., Lezec, H.J., Ghaemi, H.F., Thio, T. \& Wolff, P.A. (1998) Extraordinary optical transmission through sub-wavelength hole arrays. Nature. 391, 667-669.

Elsner, H. \& Meyer, H. (2001) Nanometer and high aspect ratio patterning by electron beam lithography using a simple DUV negative tone resist. Microelectron. Eng. 57-58, 291-296. 
Grosjean, T. \& Courjon, D. (2003) Polarization filtering induced by imaging systems: effect on image structure. Phys. Rev. E 67(4), 46611.

Kanamori, Y., Shimono, M. \& Hane, K. (2006) Fabrication of transmission color filters using silicon subwavelength gratings on quartz substrates. IEEE Photon. Technol. Lett. 18(20), 2126-2128.

Van Labeke, D. \& Barchiesi, D. (1993) Probes for scanning tunneling optical microscopy: Theoretical comparison. J. Opt. Soc. Am. A 10(10), 21932201.

Liu, Y. \& Blair, S. (2003) Fluorescence enhancement from an array of subwavelength metal apertures. Opt. Lett. 28(7), 507-509.

Martin-Moreno, L., Garcia-Vidal, E., Lezec, H., Pellerin, K., Thio, T., Pendry, J. \& Ebbesen, T. (2001) Theory of extraordinary optical transmission through subwavelength hole arrays. Phys. Rev. Lett. 86, 11141117.

Mussot, A., Sylvestre, T., Provino, L. \& Maillotte, H. (2003) Generation of a broadband single-mode supercontinuum in a conventional dispersion shifted fiber by use a nanosecond microchip laser. Opt. Lett. 28(19), 1820-1822.
Perentes, A., Utke, I., Dwir, B. et al. (2005) Fabrication of arrays of subwavelength nano-apertures in an optically thick gold layer on glass slides for optical studies. Nanotechnology. 16, S273-S277.

Popov, E., Neviere, M., Enoch, S. \& Reinisch, R. (2000) Theory of light transmission through subwavelength periodic hole arrays. Phys. Rev. B 62, 16100-16108.

Poujet, Y., Roussey, M., Salvi, J., Baida, F., Van Labeke, D., Perentes, A., Santschi, C. \& Hoffmann, P. (2006) Super-transmission of light through subwavelength annular aperture arrays in metallic films: spectral analysis and near-field optical images in the visible range. Photonics Nanostruct.: Fundam. Appl. 4, 47-53.

Roberts, A. \& McPhedran, R. (1988) Bandpass grids with annular apertures. IEEE Trans. Antennas Propag. 36, 607.

Salvi, J., Roussey, M., Baida, et al. (2005) Annular aperture arrays: study in the visible range of the electromagnetic spectrum. Opt. Lett. 30, 16111613.

Shinada, S., Hashizume, J. \& Koyama, F. (2003) Surfaceplasmon resonance on microaperture vertical-cavity surface-emitting laser with metal grating. Appl. Phys. Lett. 83(5), 836-838. 